\title{
X-ray Crystal Structure Determination of LTA4H:4MDM:PGP Analogue Complex and Characterization of the Aminopeptidase Enzyme Mechanism
}

\author{
Kyung Hyeon Lee, ${ }^{\mathrm{a}, \mathrm{b}}$, Soo Hyeon Lee ${ }^{\mathrm{a}}$, Marie Burdick ${ }^{\mathrm{c}}$, Zhimin Zheng ${ }^{\mathrm{c}}$, Y. Michael \\ Shim ${ }^{\mathrm{c}}$, Mikell Paige ${ }^{\mathrm{b}}$, Schroeder M. Noble ${ }^{\mathrm{a}}$ \\ a Department of Wound Infections, Bacterial Diseases Branch, Walter Reed Army \\ Institute of Research, 503 Robert Grant Avenue, Silver Spring, MD 20910, \\ schroeder.m.noble.civ@mail.mil \\ ${ }^{\mathrm{b}}$ Department of Chemistry \& Biochemistry, George Mason University, Manassas, VA \\ 20110,mpaige3@gmu.edu \\ ${ }^{\mathrm{c}}$ Division of Pulmonary \& Critical Care Medicine, Department of Medicine, University of \\ Virginia, Charlottesville, VA 22908, yss6n@hscmail.mcc.virginia.edu
}

The zinc metalloenzyme leukotriene $\mathrm{A}_{4}$ hydrolase $\left(\mathrm{LTA}_{4} \mathrm{H}\right)$ participates in both proinflammatory and anti-inflammatory pathways $[1,2]$. It functions as an epoxide hydrolase (EH) in pro-inflammatory pathways and an aminopeptidase (AP) in antiinflammatory pathways $[3,4]$. The EH activity of LTA4H is associated with LTB4mediated inflammation, while the AP activity of the enzyme was shown to promote resolution of pulmonary inflammation by clearance of the chemotactic tripeptide PGP [5, 6]. To understand the kinetic mechanisms of PGP hydrolysis, we developed an assay to quantify PGP degradation by selectively derivatizing the primary amine of Gly-Pro produced from $N$-terminal hydrolysis of PGP using fluorescamine. The LTA4H enzyme exhibited substrate-induced inhibition with escalating concentrations of the substrate, PGP [7]. Our assay showed that the $\mathrm{LTA}_{4} \mathrm{H}$ modulator, 4-methoxydiphenymethane (4MDM), rescued AP activity in the presence of PGP to $100 \mathrm{nM}$, and then failed to prevent inhibition by PGP at high concentrations. To further understand this phenomenon, we determined the first X-ray crystal structure $\mathrm{LTA}_{4} \mathrm{H}$ in complex with 4MDM and a non-hydrolysable analog of PGP at $2.8 \AA$ resolution. The PGP analog and $4 \mathrm{MDM}$ were both bound in each of the 3 molecules of $\mathrm{LTA}_{4} \mathrm{H}$ in the asymmetric unit. The structure revealed that the PGP analog and 4MDM bind within the $\mathrm{LTA}_{4} \mathrm{H}$ active site, but at a significant distance of $>4.0 \AA$ away from each other. The PGP analog bound in the AP active site with its prolyl carbonyl interacting with the catalytic zinc. 4MDM bound in the hydrophobic portion of the $\mathrm{LTA} 4 \mathrm{H}$ binding pocket in a similar orientation as the LTA4H:4MDM complex structure, and was stabilized by van der Waals interactions. Previously we reported the structure of $\mathrm{LTA}_{4} \mathrm{H}$ in complex with 4-OMeARM1, a hybrid 4MDM-ARM1 analogue. In this structure, the methoxy group of 4OMe-ARM1 interacted with the main-chain carbonyl of Q136 and limited the rotational freedom of this residue. Q136 maintained rotational freedom in the LTA4H:4MDM:PGP analog structure without interfering with the catalytic water binding to E296. The LTA4H:4MDM:PGP analog structure is not suggestive of substrate induced inhibition. Structural studies of $\mathrm{LTA}_{4} \mathrm{H}$ in complex with the PGP analog are in-progress to provide insight on the PGP assay study results. In conclusion, we have demonstrated limited potentiation of $\mathrm{LTA}_{4} \mathrm{H}$ AP activity modulated by $4 \mathrm{MDM}$ in the presence of the substrate PGP, and determined the crystal structure of $\mathrm{LTA}_{4} \mathrm{H}$ in complex with $4 \mathrm{MDM}$ and the 
PGP analog. This structural information will aid in the next round of design and synthesis of selective $\mathrm{LTA}_{4} \mathrm{H}$ AP activators.

\section{References}

[1] Jiang, X. et al. (2008) Bioorg \& Med Chem Letters, 18, 6549-6552.

[2] De Oliveira, E. O. et al. (2011) Bioorg \& Med Chem Letters, 21, 6746-6750.

[3] Stsiapanava, A. et al. (2014) Proc. Natl. Acad. Sci. U. S. A. 111, 4227-4232.

[4] Paige, M. et al. (2014) Journal of Immunology, 192, 5059-5068.

[5] Snelgrove, R. J. et al. (2010) Science, 330, 90-94.

[6] Numao, S. et al. (2017) Scientific Reports, 7, 13591.

[7] Baici, A. (2015) Springer Berlin Heidelberg, 209-288. 